\title{
New Properties of Reactive Elements and the Problem of Propagation of Electrical Signals in Long Lines
}

\author{
F.F. Mende* \\ B.I. Verkin Institute for Low Temperature Physics and Engineering NAS, Ukraine, 47 Lenin Ave., Kharkov, Ukraine \\ *Corresponding author: mende_fedor@mail.ru
}

Received September 25, 2014; Revised October 13, 2014; Accepted October 15, 2014

\begin{abstract}
Are examined the laws of the self-induction of capacitance and inductance, which it is customary to assume as reactive elements. It is shown that with the connection to the sources of direct current and voltage they have the effective resistance, which depends on the time. Is examined the parametric self-induction of the elements indicated and it is shown that in the regime of parametric self-induction the capacitance and inductance can not only derive energy from the power sources, but also return it into the external circuits. The velocity of propagation of constant voltages and currents in the long lines is obtained. In this case the laws of parametric self-induction are used. This task cannot be solved, using standard wave equations for the long lines.
\end{abstract}

Keywords: self-induction, capacitance, inductance, linear capacitance, running inductance, resistance, wave equations, Ohm law, Maxwell equations

Cite This Article: F.F. Mende, "New Properties of Reactive Elements and the Problem of Propagation of Electrical Signals in Long Lines." American Journal of Electrical and Electronic Engineering, vol. 2, no. 5 (2014): 141-145. doi: 10.12691/ajeee-2-5-1.

\section{Introduction}

Many specialists consider that the capacitance and inductance are reactive elements and cannot take away energy in the alternating current circuits or voltage [1,2]. In order to be convinced of the fact that this is not always carried out, it suffices to conduct simple experiment. If we connect capacitor to the electric brush of alternating current, to and then open it, then it is possible to reveal that the capacitor is charged. If we repeat this experiment repeatedly, then it is possible to establish that a voltage drop across the terminals of capacitor varies in the limits from the zero values, to the amplitude value of a potential difference in the network. This fact attests to the fact that the capacitor can take away energy from the electric brush, since the energy, accumulated in the capacitor, is determined by the equation of

$$
W_{C}=\frac{1}{2} C U^{2}
$$

where of $U$ - voltage drop across the terminals of capacitor.

To load capacitor is possible and from the source of direct current, and in this case the energy, accumulated in the capacitor, will be obtained from this source. Idea about the fact that the reactive elements cannot take away energy in the alternating current circuits, was formed for that reason, that with the examination of the properties of reactive elements in such chains are examined the periodic processes of infinite duration and the moment of turning off of reactive element from the chain is not considered.
If we charge capacitor from the source of direct current, then a voltage drop across it will be grow, and it will derive energy from the source, accumulating it similar to storage battery. In this case the capacitance can be represented as the effective resistance, which depends on the time. The same relates also to the inductance, connected to the dc power supply. But capacitance and inductance can return energy into the external circuit, if their value changes.

The special features of capacitance and inductance indicated make it possible to solve a question about the velocity of propagation of constant voltage and currents in the long lines, what cannot be made, using telegraphic of equations.

\section{Laws of the Self-induction}

To the laws of self-induction should be carried those laws, which describe the reaction of such elements of radio-technical chains as capacitance, inductance and resistance with the galvanic connection to them of the sources of current or voltage. These laws are the basis of the theory of electrical chains. The results of this theory can be postponed also by the electrodynamics of material media, since such media can be represented in the form equivalent diagrams with the use of such elements.

By self-induction we will understand the reaction of material structures with the constant parameters to the connection to them of the sources of voltage or current. To the self-induction let us carry also that case, when its parameters can change with the presence of the connected power source or the energy accumulated in the system. This self-induction we will call parametric. 
Let us refine some terms, utilized in the article. We will call such generator, which ensures lumped voltage on any external circuit constant-potential generator. The internal resistance of the ideal voltage generator is equal to zero. We will call such generator, which provides the assigned current in any external circuit direct-current generator. The internal resistance of ideal current generator approaches infinity.

Ideal capacitance cannot be connected to the ideal voltage source, since at the moment of connection the current of the charge of capacitance will approach infinity. Capacitance can be connected only to the current source. Ideal inductance cannot be connected to the ideal current source, since. at the moment of connection a voltage drop across inductance will approach infinity. Inductance can be connected only to the dc power supply.

\section{Capacitive Self-induction}

If the capacitance of $C$ is charged to a potential difference of $U$, then the charge of $Q$, accumulated in it, is determined by the equation of

$$
Q_{C, U}=C U \text {. }
$$

Magnitude of the charge can change with the method of changing the potential difference with a constant capacitance, or with a change in the capacitance with a constant potential difference.

If capacitance value or voltage drop across it depend on time, then the current strength is determined by the equation

$$
I=\frac{d Q_{C, U}}{d t}=C \frac{\partial U}{\partial t}+U \frac{\partial C}{\partial t} . .
$$

Consequently, current in circuit can be obtained by two methods: changing voltage on the capacitance with a constant capacitance or changing capacitance with constant voltage on it.

For the case, when the capacitance of $C_{0}$ is constant, we obtain

$$
I=C_{0} \frac{\partial U}{\partial t} .
$$

Let us connect to the capacitance direct-current generator and we will support in it the direct current of $I_{0}$. Then, after integrating Eq. (3.2) with respect to the time, we obtain

$$
U=\frac{I_{0} t}{C_{0}} .
$$

This equation, which connects the direct current, which flows through the capacitance and voltage on it is the analog of Ohm law. For this reason the value of

$$
R=\frac{t}{C_{0}}
$$

is effective resistance, which depends on time. The it should be noted that obtained result is completely obvious; however, such properties of capacitance, which customary to assume by reactive element they were for the first time noted in the work [3].
The power, output by current source, is determined in this case by the equation

$$
P(t)=\frac{I_{0}{ }^{2} t}{C_{0}} .
$$

The energy, accumulated by capacitance in the time, we will obtain, after integrating Eq. (3.4) with respect to the time

$$
W_{C}=\frac{I_{0}^{2} t^{2}}{2 C_{0}} .
$$

Substituting here the value of current from Eq. (3.3), we obtain the dependence of the value of the accumulated in the capacitance energy from the instantaneous value of voltage on it

$$
W_{C}=\frac{1}{2} C_{0} U^{2} .
$$

If we support at the capacitance constant voltage of $U_{0}$, and to change capacitance, then the current, which flows through it, is written down

$$
I=U_{0} \frac{\partial C}{\partial t} .
$$

This case relates to the parametric self-induction. This equation, which connects the direct current, which flows through the capacitance and voltage on it is the analog of Ohm law. For this reason the value of

$$
R_{C}=\left(\frac{\partial C}{\partial t}\right)^{-1}
$$

is effective resistance, which depends on time.

The power, expended in this case by source, is determined by the equation:

$$
P=U_{0}^{2} \frac{\partial C}{\partial t} .
$$

From Eq. (3.6) is evident that depending on the sign of derivative the expendable power can have different signs. When the derived positive, expended by source power proceeds with an increase in the energy, stored up in the capacitance. If derived negative, then work carries out the external source, which ensures the decrease of capacitance. The energy, spent by this source, is separated in the external circuit.

Let us examine one additional process, which falls under for the determination of capacitive self-induction. From Eq. (3.1) it is evident that if the charge, accumulated in the capacity, remains constant, then stress on it can be changed by changing the capacity. In this case is fulfilled the equation

$$
C U=C_{0} U_{0}=\text { const, }
$$

$C$ and $U$ - instantaneous values, $C_{0}$ and $U_{0}$ - initial values of these parameters.

The stress on the capacity and the energy, accumulated in it, will be in this case determined by the equations

$$
U=\frac{C_{0} U_{0}}{C}
$$




$$
W_{C}=\frac{1}{2} \frac{\left(C_{0} U_{0}\right)^{2}}{C} .
$$

This process of self-induction is connected with a change in the capacity. Therefore it includes the determination of parametric self-induction.

\section{Inductive Self-induction}

Let us examine the processes, proceeding in the inductance. Let us introduce the concept of the flow of

$$
\Phi_{L, I}=L I .
$$

Voltage on the inductance is equal to the derivative of the flow of current induction on the time:

$$
U=\frac{d \Phi_{L, I}}{d t}=L \frac{\partial I}{\partial t}+I \frac{\partial L}{\partial t} .
$$

Let us examine the case, when the inductance of is constant.

$$
U=L_{0} \frac{\partial I}{\partial t} .
$$

We will support in it constant voltage of $U_{0}$. After integrating Eq. (4.1) with respect to the time, we obtain

$$
I=\frac{U_{0} t}{L_{0}} .
$$

This equation, which connects the direct current, which flows through the capacitance, and voltage on it is the analog of Ohm's law. Therefore the value of

$$
R=\frac{L_{0}}{t}
$$

plays the role of effective resistance, which depends on time.

The power, expended in this case by source, is determined by the equation

$$
P(t)=\frac{U_{0}{ }^{2} t}{L_{0}} .
$$

This power linearly depends on time. After integrating Eq. (4.2) on the time, we will obtain the energy, accumulated in the inductance of

$$
W_{L}=\frac{1}{2} \frac{U^{2} t^{2}}{L} .
$$

After substituting in this equation the value of voltage from Eq. (4.2), we obtain the energy, stored up in the inductance,

$$
W_{L}=\frac{1}{2} L_{0} I^{2} .
$$

If we support in the inductance the direct current of $I_{0}$, and to change inductance, then the current, which flows through it, will be written down

$$
U=I_{0} \frac{\partial L}{\partial t} .
$$

This case relates to the parametric self-induction. This equation, which connects the direct current, which flows through the capacitance and voltage on it is the analog of Ohm's law. Therefore the value of

$$
R=\frac{d L}{d t}
$$

is the effective resistance.

The power, expended in this case by source, is determined by the equation

$$
P=I_{0}^{2} \frac{\partial L}{\partial t} .
$$

As in the case with the capacitance, effective resistance can be both the positive and negative. This means that the inductance can how derive energy from without, so also return it into the external circuits.

If inductance is made from superconductor and is bridged, then

$$
\Phi=L_{1} I_{1}=\text { const },
$$

$L_{1}$ and $I_{1}$ - initial values of these parameters, which are located at the moment of the short circuit of inductance with the presence in it of current.

This regime we will call the regime of the frozen flow. In this case the equation is fulfilled

$$
I=\frac{I_{1} L_{1}}{L},
$$

$I$ and $L$ - the instantaneous values of the corresponding parameters.

In flow regime examined of current induction remains constant, however, in connection with the fact that current in the inductance it can change with its change, this process falls under for the determination of parametric self-induction. The energy, accumulated in the inductance, in this case will be determined by the equation

$$
W_{L}=\frac{1}{2} \frac{\left(L_{1} I_{1}\right)^{2}}{L} .
$$

If inductance is made from superconductor and is bridged, then

$$
\Phi=L_{1} I_{1}=\text { const },
$$

$L_{1}$ and $I_{1}$ - initial values of these parameters, which are located at the moment of the short circuit of inductance with the presence in it of current.

This regime we will call the regime of the frozen flow. In this case the equation is fulfilled

$$
I=\frac{I_{1} L_{1}}{L},
$$

$I$ and $L$ - the instantaneous values of the corresponding parameters.

In flow regime examined of current induction remains constant, however, in connection with the fact that current in the inductance it can change with its change, this process falls under for the determination of parametric self-induction. The energy, accumulated in the inductance, in this case will be determined by Eq. 


$$
W_{L}=\frac{1}{2} \frac{\left(L_{1} I_{1}\right)^{2}}{L}
$$

\section{Propagations of Constant Voltage and Current in the Long Lines}

The processes of the propagation of voltages and currents in the long lines determine the wave equations

$$
\begin{aligned}
& \frac{\partial^{2} U}{\partial z^{2}}=\frac{1}{v^{2}} \frac{\partial^{2} U}{\partial t^{2}}, \\
& \frac{\partial^{2} I}{\partial z^{2}}=\frac{1}{v^{2}} \frac{\partial^{2} I}{\partial t^{2}},
\end{aligned}
$$

which are obtained from the equations of telegraphy

$$
\begin{aligned}
& \frac{\partial U}{\partial z}=-L_{0} \frac{\partial I}{\partial t}, \\
& \frac{\partial I}{\partial z}=-C_{0} \frac{\partial U}{\partial t},
\end{aligned}
$$

$L_{0}$ and $C_{0}$ linear inductance and the capacitance of line.

The velocity of propagation in this line is determined by the equation

$$
v=\frac{1}{\sqrt{L_{0} C_{0}}}
$$

The knowledge second derivative voltages and currents is required during the solution of wave equations.

But wave equations do not give the answer to the question, as one should enter when to incoming line it is connected dc power supply. The results, obtained in two previous divisions, give answer to these questions. The processes, examined in two previous paragraphs, concern chains with the lumped parameters, when the distribution of potential differences and currents in the elements examined can be considered uniform. However, there are chains, for example the long lines, in which the spatial distribution of voltages and currents they are not uniform.

Let us examine processes in the long line, whose capacitance and inductance are the distributed parameters. We will consider that the linear capacitance and the inductance of line is equal $C_{0}$ and $L_{0}$ respectively. If we to this line connect the dc power supply of $U_{0}$, then its front will be extended in the line some by the speed of $v$, and the moving coordinate of this front will be determined by the equation of $z=v t$. In this case the total quantity of the charged capacitance and the value of the summary inductance, along which it flows current, calculated from the beginning lines to the location of the front of voltage, will change according to the law

$$
\begin{aligned}
& C(t)=z C_{0}=v t C_{0}, \\
& L(t)=z L_{0}=v t L_{0} .
\end{aligned}
$$

The source of voltage of will in this case charge the being increased capacitance of line, for which from the source to the charged line in accordance with Eq. (3.5) must leak the current

$$
I=U_{0} \frac{\partial C(t)}{\partial t}=v U_{0} C_{0}
$$

This current there will be the leak through the conductors of line, that possess inductance. But, since the inductance of line in connection with the motion of the front of voltage, also increases, in accordance with Eq. (4.4), on it will be observed a voltage drop

$$
U=I \frac{\partial L(t)}{\partial t}=I v L_{0}=v^{2} U_{0} C_{0} L_{0} .
$$

But a voltage drop across the conductors of line in the absolute value is equal to the voltage, applied to its entrance; therefore in the last expression should be placed $U=U_{0}$. From Eq. (5.1) we obtain

$$
v=\frac{1}{\sqrt{L_{0} C_{0}}} .
$$

This equation coincides with the velocity of propagation, obtained from the wave equations.

However, in the electrodynamics, based on Maxwell's equations, there are no such concepts as capacitance and inductance, and there are concepts of the electrical and magnetic permeability of medium. In the carried out examination such concepts as electrical and magnetic fields also was absent. Let us show how to pass from such categories as linear inductance and capacitance, current and voltage in the line to such concepts as dielectric and magnetic constant, and also electrical and magnetic field. For this let us examine the simplest construction of line, located in the vacuum (Figure 1)

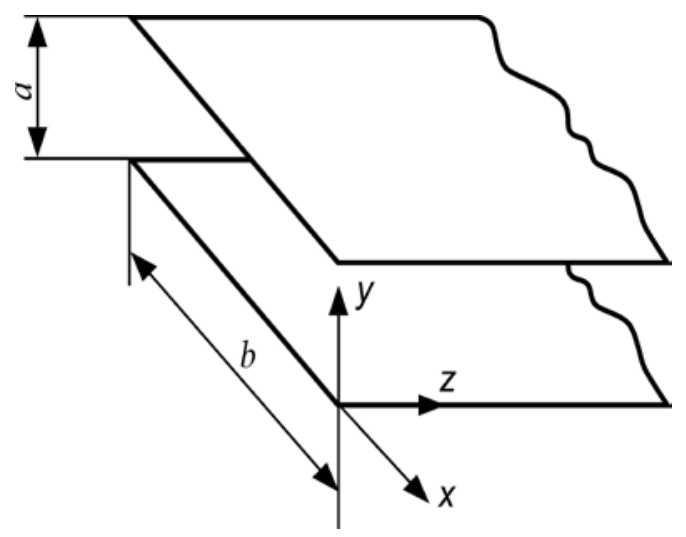

Figure 1. The two-wire circuit, which consists of two ideally conducting planes

We will consider that $b>>a$ and edge effects it is possible not to consider. Then the following connection will exist between the linear parameters of line and the magnetic and dielectric constants

$$
\begin{aligned}
& L_{0}=\mu_{0} \frac{a}{b}, \\
& C_{0}=\varepsilon_{0} \frac{b}{a},
\end{aligned}
$$

$\mu_{0}$ and $\varepsilon_{0}$ - dielectric and magnetic constant of vacuum.

The velocity of propagation in this line is determined by the equation 


$$
v=\frac{1}{\sqrt{L_{0} C_{0}}}=\frac{1}{\sqrt{\mu_{0} \varepsilon_{0}}}=c,
$$

$c$ - velocity of propagation of light in the vacuum.

The surge impedance of the line examined will be equal

$$
Z=\frac{a}{b} \sqrt{\frac{\mu_{0}}{\varepsilon_{0}}}=\frac{a}{b} Z_{0},
$$

$Z_{0}=\sqrt{\frac{\mu_{0}}{\varepsilon_{0}}}$ - surge impedance of free space.

This with the observance of the condition of $a=b$ we obtain the equality of $L_{0}=\mu_{0}$.This means that magnetic permeability of $\mu_{0}$ plays the role of the longitudinal specific inductance of vacuum. In this case is observed also the equality of $C_{0}=\varepsilon_{0}$. This means that the dielectric constant of $\varepsilon_{0}$ plays the role of the transverse specific capacitance of vacuum. In this interpretation both $\mu_{0}$ and $\varepsilon_{0}$ acquire clear physical sense.

If we to the line of infinite length, connect the dc power supply of $U_{0}$, then the field strength in the line will be equal

$$
E_{y}=\frac{U_{0}}{a},
$$

and the current, which flows into the line from the power source, will be determined by the equation

$$
I=\frac{U_{0}}{Z}=\frac{a E_{y}}{Z} .
$$

Magnetic field in the line will be equal to the specific current, flowing in the line

$$
H_{X}=\frac{I}{b}=\frac{a E_{y}}{b Z} .
$$

Substituting here the value of $Z$, we obtain

$$
H_{x}=\frac{E_{y}}{Z_{0}} .
$$

The same connection between the electrical and magnetic field exists also for the case of the transverse electromagnetic waves, which are extended in the free space.

Comparing expressions for the energies, it is easy to see that the specific energy can be expressed through the electrical and magnetic fields

$$
\frac{1}{2} \mu_{0} H_{x}^{2}=\frac{1}{2} \varepsilon_{0} E_{y}^{2} .
$$

This means that the specific energy, accumulated in the magnetic and electric field in this line is identical.

The special feature of this line will be the fact that in it, in contrast to the free space, the stationary magnetic and electric fields can be extended, but this case cannot be examined by the method of solution of Maxwell's equations.

Consequently, conditionally it is possible to consider that the long line is the device, which with the connection to it of dc power supply is filled up with two forms of the energy: electrical and magnetic. The specific densities of these energies are equal, and since and electrical and magnetic energy fill identical volumes, the general energy, accumulated in these fields is identical. The distribution of electrical and magnetic pour on in the section from the beginning of line and to the front of voltage in it is uniform.

Is obvious that the work of $I(t) U(t)$ represents the power of $P$, transferred through the cross section of line in the direction of $z$. If in this equation current and voltage was replaced through the tensions of magnetic and electrical pour on, then we will obtain $P=a b E_{y} H_{x}$. The work of $E_{y} H_{x}$ represents the absolute value of Poynting vector.

\section{Conclusion}

In the article are examined the laws of the selfinduction of reactive elements. It is shown that such reactive elements as capacitance and inductance with their connection to the current generators and voltage present the effective resistance, whose value depends on time. Before the appearance of work [3] about such properties of these elements it was not known. Is introduced the concept of the parametric induction of reactive elements, which occurs when the capacitance value or inductance it changes. Introduction of this concept made possible to calculate the velocity of propagation of constant voltages and currents in the long lines, what cannot be made with the use of wave equations.

\section{References}

[1] S. Ramo, J. Uinneri. Fields and wave in contemporary radio engineering. OGIZ, 1948.

[2] I. S. Gonorovskiy. Radio-technical chains and the signal. M,: Soviet radio, 1977.

[3] F. F. Mende. Great errors and the error of the physicists of XIX$X X$ of centuries. Revolution in contemporary physics. Kharkov, NTMT, 2008. 